\title{
Discussion of the Effect of Positional Tolerance on other Tolerances Shu-ping LV
}

\author{
Dalian Vocational \& Technical College, Dalian, China
}

Keywords: dimension tolerance zone, position tolerance zone, coaxiality, MMC status

\begin{abstract}
For geometrical tolerance projects, some tolerance projects relate to other geometrical tolerances, namely a individual geometrical tolerance can control several other geometrical tolerances. If we can not accurately judge the related geometrical tolerance which can be natural controlled by a certain geometrical tolerance, it will lead to the excess phenomenon or tolerance value contradiction phenomenon of the geometrical tolerances. Position tolerance is the most typical tolerance that can combined control other tolerances. This paper mainly describes how to use the position tolerance to control the dimensional tolerance and coaxiality tolerance, so that everyone can make a selection in the specific application.
\end{abstract}

\section{The Combined Control of Dimension Tolerance and Position}

Function of Dimension Tolerance Zone. As show in Fig.1, it is the requirement of dimension and position tolerance for a work piece, then how to confirm the holes of $3-\phi D$ are up to specification, the analysis as followed.

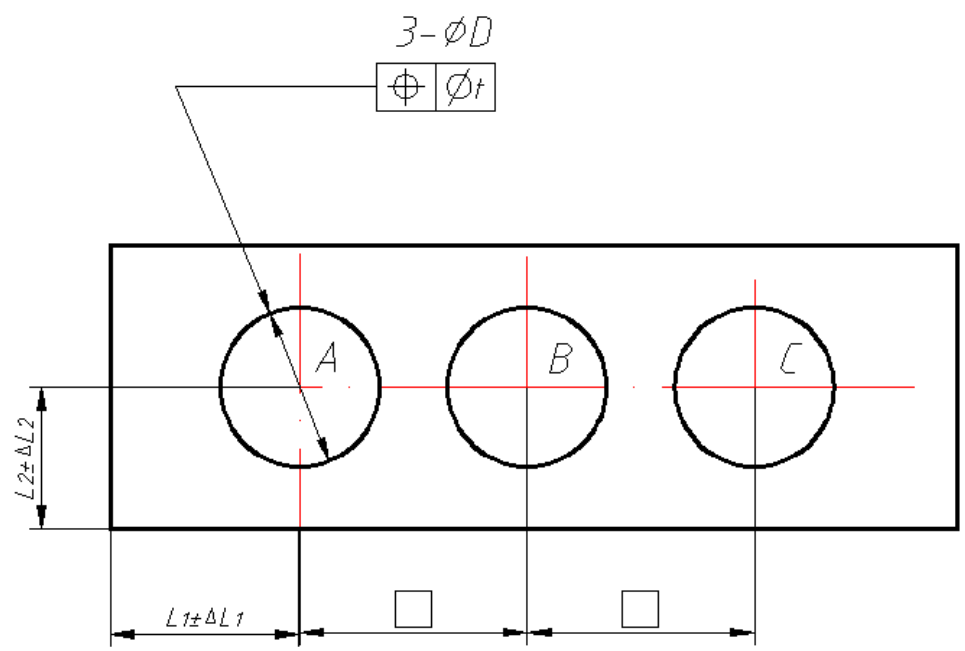

Fig.1 Tolerance requirement of a work piece

As show in Fig.2, dimension tolerance zone $2 \Delta l_{1}$ only restricts the position of hole $A$ in $X$ direction, but the direction and position of hole $B, C$ will not be restricted. The position in $X$ direction of these two holes was controlled by position. Dimension tolerance zone $2 \Delta l_{2}$ restrict the of hole $A, B, C$ in $Y$ direction, then the axis of actual hole will in the dimension tolerance zone.

Function of Position Tolerance Zone. According to the type of the measured features, the position is divided into the position of point, the position line and the position of plane. This example is the position of the hole axis.

As show in Fig.2, the position tolerance zone of hole can confirm the axis of the actual hole. The part of position tolerance zone which exceeding the dimension tolerance zone will be invalid. After locating the position tolerance zone of hole A (the first hole), other holes' positions will be confirmed by theoretically exact dimension between holes. According to without group holes location datum, its' geometry frame will horizontal and vertical move or rotate to determine the position of each hole's position tolerance zone. 


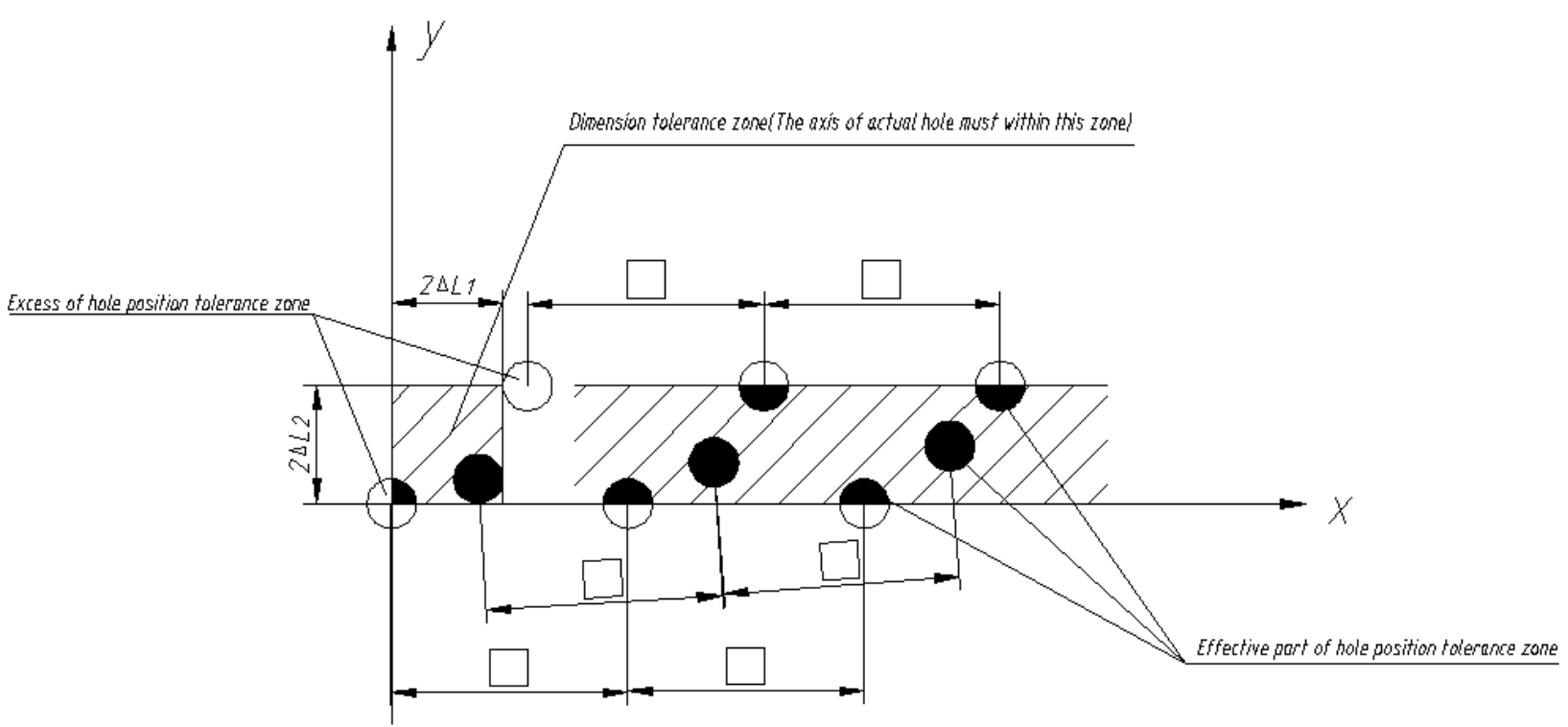

Fig.2 Relationship between dimension tolerance zone and position tolerance zone

Combined Control of Position Tolerance Zone and Dimension Tolerance Zone. The position of actual holes' features, namely, the position of axis for actual hole is in the coincidence part between dimension tolerance zone and position tolerance zone. Although the axis of actual hole was controlled by position tolerance zone, the range of position tolerance zone which exceeding the dimension tolerance zone is invalid. So long as the errors of actual features are in the position tolerance zone of the hole, or in the allowable value tolerance zone, it will be qualified.

For example, there is a plate with three holes, the requirement is not only maintaining the correct position between these three holes, but also locating the correct geometric frame of the hole-group. So the geometrical frame of this hole-group must be confirmed by theoretically exact dimension. As show in Fig. 3, the position of the hole in the MMC status is $\phi 0.4 \mathrm{~mm}$, Obey the measured error of each hole, we can confirm whether the hole is qualified.

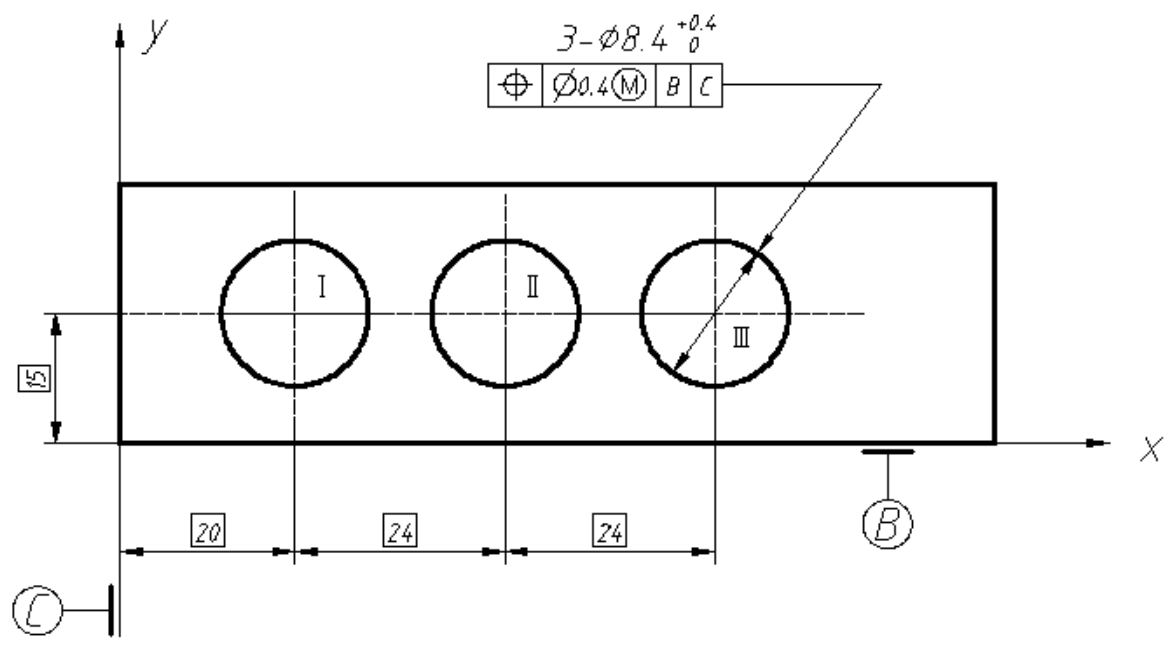

Fig.3 The hole group is located by theoretically exact dimension

First, the hole group is located by theoretically exact dimension, so the tolerance zone of this hole group is zero. The ideal position center of the hole-group is unique and fixed, and it does not affect the calculation of the position error.

Second, during the process of calculate the errors, we will start from datum plane $B, C$ to measure the actual diameter dimension of these three holes, and the measured results data are shown in Table 1. 
Table 1. Measured results data

\begin{tabular}{|c|c|c|c|c|c|c|c|c|c|c|}
\hline \multirow[t]{2}{*}{ No. } & \multicolumn{3}{|c|}{ Actual measurement } & \multicolumn{3}{|c|}{ Error } & \multicolumn{3}{|c|}{ Tolerance } & \multirow[b]{2}{*}{ Result } \\
\hline & $\begin{array}{l}x \\
\text { directio } \\
n\end{array}$ & $\begin{array}{l}Y \\
\text { Direction }\end{array}$ & $\begin{array}{l}\text { Diamete } \\
r\end{array}$ & $f x$ & fy & $\mathrm{f}$ & $t e+$ & $t B=$ & & \\
\hline I & 19.8 & 14.8 & $\Phi 8.6$ & $\begin{array}{l}-0 . \\
2 . \\
+0 .\end{array}$ & $\begin{array}{l}-0 . \\
2 \\
-0 .\end{array}$ & $\begin{array}{l}0.28 \\
3 \\
0.35\end{array}$ & $\begin{array}{l}\Phi \\
0.4 \\
\Phi\end{array}$ & $\Phi 0.2$ & $\Phi 0.6$ & Good \\
\hline II & 44.2 & 14.7 & $\Phi 8.6$ & $\begin{array}{l}2 \\
+0 .\end{array}$ & $\begin{array}{l}3 \\
+0 .\end{array}$ & $\begin{array}{l}1 \\
0.14\end{array}$ & $\begin{array}{l}0.4 \\
\Phi\end{array}$ & $\Phi 0.2$ & $\Phi 0 . \overline{6}$ & NG \\
\hline III & 58.1 & 15.1 & $\Phi 8.6$ & 1 & 1 & 1 & 0.4 & $\Phi 0.2$ & $\Phi 0.6$ & Good \\
\hline
\end{tabular}

Remark: Error $f>\frac{t_{G}}{2}$ or $>\frac{t_{Y}}{2}$ is out of tolerance.

By calculate method: Suppose the error $f$ is the Vector sum of $f_{x}$ and $f_{y}$, the datum plane $B$ and datum plane $C$ is vertical.

So

$$
f=\sqrt{f_{x}^{2}+f_{y}^{2}}
$$

Take the hole II for an example to calculate the errors. According to the data that the diameter of the hole is $8.6 \mathrm{~mm}$, the largest entity has deviated $0.2 \mathrm{~mm}$, this is the compensation value. The measured data $x, y$ coordinates were $44.2 \mathrm{~mm}$ and $14.7 \mathrm{~mm}$, so the distance (or error) between the actual measurement point and the theoretically center position which determined by the distance of $44 \mathrm{~mm}$ and $15 \mathrm{~mm}$ is:

$$
\left\{\begin{array}{l}
f_{x}=44.2-44=0.2 \\
f_{y}=14.7-15=-0.3
\end{array}\right.
$$

So

$$
f_{\Pi}=\sqrt{0.2^{2}+(-0.3)^{2}}=\sqrt{0.13}=0.361
$$

Evaluation result is as follows:

$$
\left\{\begin{array}{l}
0.361>\frac{\phi 0.4}{2} \text { and } 0.361>\frac{\phi 0.6}{2} \\
f_{\Pi}>\frac{t_{Y}}{2}>\frac{t_{G}}{2}
\end{array}\right.
$$

So it is the NG part.

The following analysis is as follows. (1) The principle of determining whether the part is qualified is mainly based on the " $t$ " of the given tolerance value. If the hole is in the deviation from the maximum physical state, according to the tolerance of the allowable value " $t$ allowed" to determine. (2)The tolerance of these three holes is within the half of the position tolerance zone ( $(\phi 0.4) / 2)$, only the hole III is not out of the tolerance, the other two holes are out of tolerance. If the compensation value is considered, the error of the hole $I$ is $0.283<0.6 / 2$, which is within the allowable value and can be considered as not out of the tolerance, but the hole II is still out of tolerance (as shown in Table 1). (3)The part is substandard for one of the hole is unqualified. As the previous introduction, it is known that the theoretically center position of the hole which determined by theoretically exact dimension and will not affect the calculate of position errors. At the same time, the graphic method will not be affected by the size also.

It can also be used to solve the distance error between the center of the hole and the theoretically center, as show in Fig. 4. 


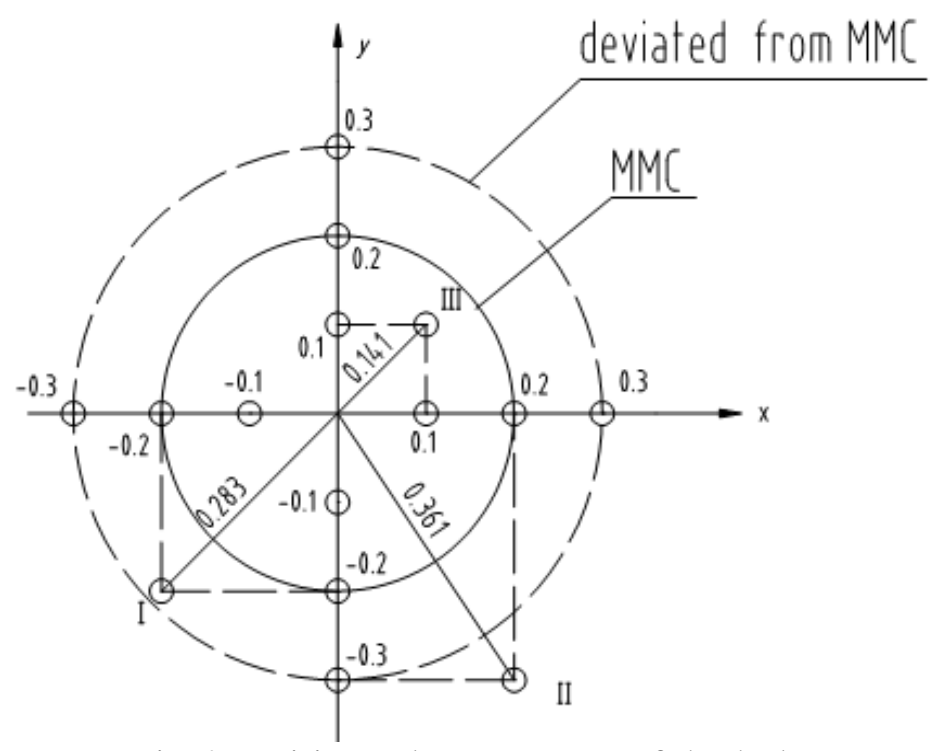

Fig.4 Position tolerance zone of the hole

\section{Position Controls Coaxial Tolerance}

Analysis of Position and Coaxial Tolerance. As usual, position can control some geometrical tolerances, for example, the parallelism can control the flatness. And the position error of some part can be controlled by different position tolerance, for instance, coaxiality of two gyration surface can be controlled by coaxial tolerance, and also can be controlled by run-out tolerance. Which position tolerance control is suitable, we should choose the appropriate one. How to choose the appropriate control method is based on the part's function and test conveniently.

Marking on the drawing is in order to test the requirement of coaxial tolerance after machining, but rotary surface will be easy to arise several Manufacturing errors, such as non-straight or non-circle errors. In addition, there is a complex factor in the slewing surface, it is difficult to find the axis of slewing center on the actual work piece. In this situation, we have to test the work piece by other form and structures which relate with axis. This indirect measurement is liable to cause comprehensive errors between the measured surface, datum plane and theoretically exact axis. So it is difficult to confirm the axis.

If it is two or more than two step-shaft (or hole), in MMC status, is favorable to adopt position to control coaxiality, and it is more convenient than using coaxiality gauge to test, as show in Fig.5.

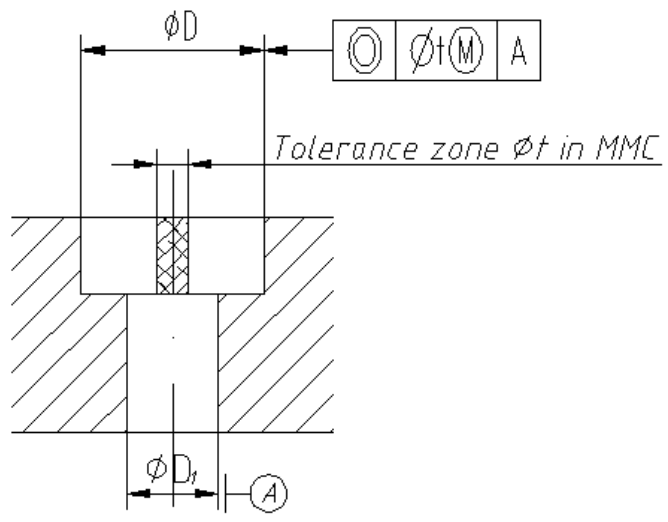

(a) axiality

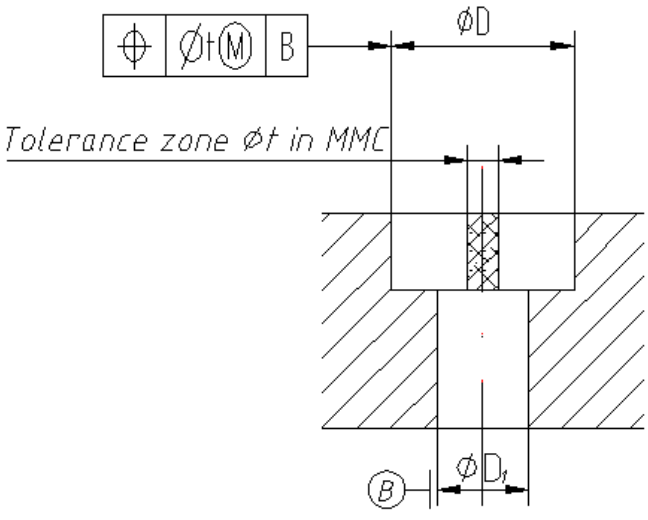

(b) positon

Fig.5 Position control coaxial tolerance

Controlling the Coaxial Hole by Position. Usually, in addition to the assembly has a high coaxiality requirement, or thin-walled parts in the processing of the fixture is easy to produce deformation, the work piece should be marked with the location of position.

Using the position tolerance to control four coaxial holes of the same size. As show in Fig.6. 

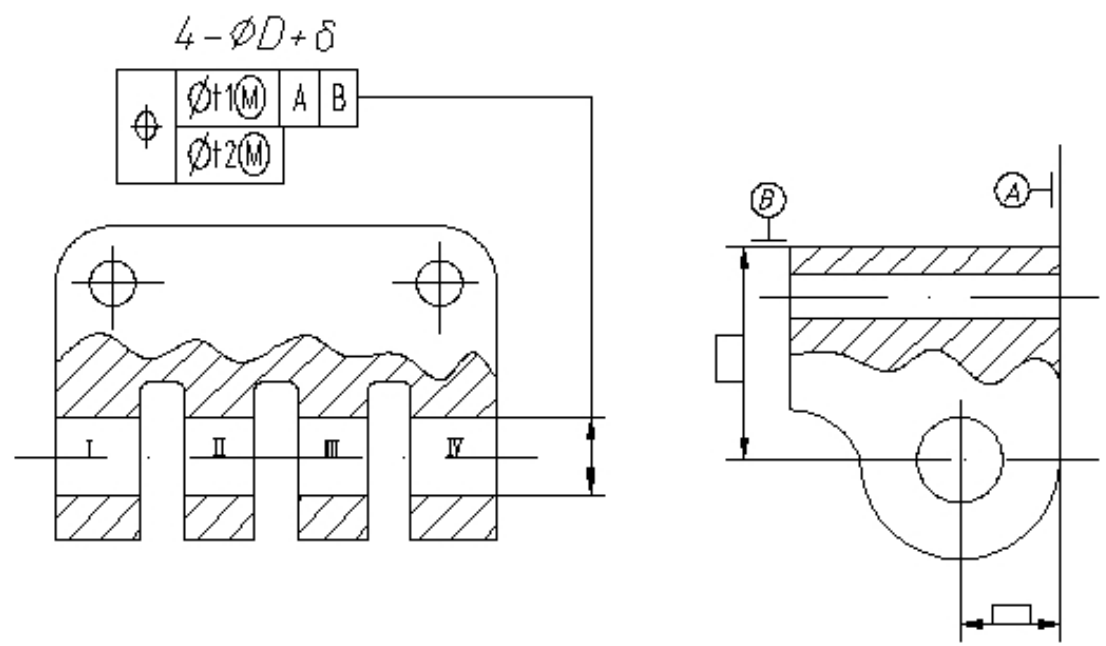

(a) position marking

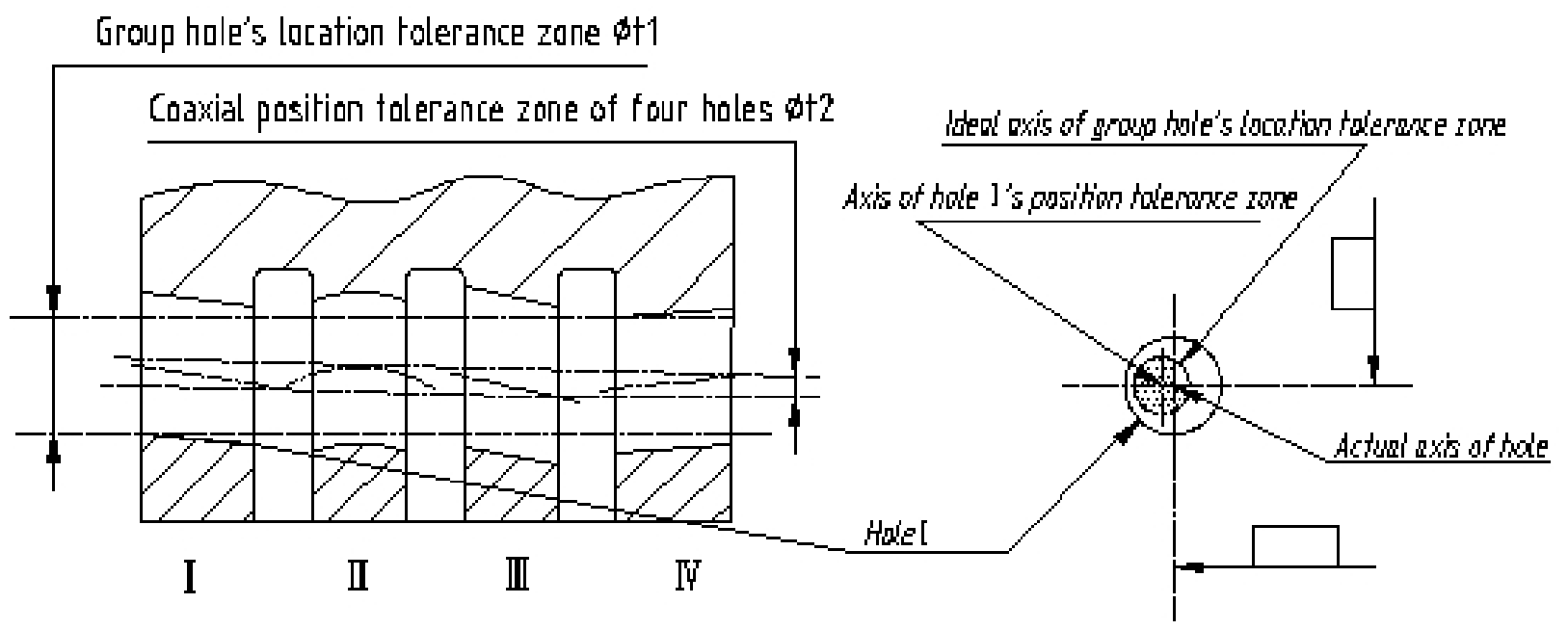

(b) Position tolerance zone

Fig.6 Position of four coaxial holes with same diameter

Then a specific example analysis as followed.

(1) The group holes and the hole are both located by theoretically exact dimension, according to the functional requirements of the parts to allow the use of composite position tolerance, as show in Fig.6(a).

(2) Group holes positioning tolerance zone: the axis of a group of four holes must be located in the four coaxial positioning tolerance zones that were located in correct position by given reference features as the base, in MMC condition, the diameter of tolerance zone is $\phi t_{1}$, as show in Fig. 6(b).

(3) Each hole tolerance zone: the axis of four holes must be located in the four interconnected coaxial position tolerance zone, in MMC condition, the diameter of tolerance is $\phi t_{2}$, as show in Fig.6 (b).

(4) In these four holes, take the hole I for an example, we can confirm the actual location of the hole I, as show in Fig.6 (b).

To be clear, Group holes' tolerance zone $\phi t_{1}$ is the ideal position to control the position tolerance of the four holes, that is the ideal axis position; the position tolerance zone of each hole $\phi t_{2}$ can control the position of axis for actual hole.

Thus it can be seen from above, there is a close correspondence between the location of work piece and the datum surface, and most of them are in accord with the concept of position. Therefore, the position has the ability to control the coaxiality and the more shape and position tolerance, so it is widely used. 


\section{Conclusion}

The geometrical tolerances are mainly based on the functional requirements of the measured features and the processing economy and the structure and rigidity of the parts. We should choose the project that simple and easy to measure, give full play to the function of the comprehensive control project, in order to reduce the geometrical tolerance items given on the drawing and the corresponding error detection project. Position, which regards the functions of the work pieces as the fundamental basis to choose the tolerance, can not only meet the requirement of production, but also reduce the cost. Therefore, Position tolerance is a popular geometrical tolerance during production, and it will be widely used.

\section{References}

[1] HUANG Y. Tolerance Control and Measurement Technology. Beijing: Machinery Industry Press, 2014.

[2] XING M.. Interchangeability and Measuring Technology. Beijing: Tsinghua University Press, 2011.

[3] XU M.. Tolerance and Fit and Technique measurement. Beijing: Machinery Industry Press, 2015.

[4] YANG T. Interchangeability and Measuring Technology. Beijing: Electronics Industry Press, 2010.

[5] SHI L. Tolerance Control and Measurement Technology. Shanghai: Fudan University Press, 2012.

[6] ZHAO Y. Tolerance and Fit and Technique measurement. Beijing: Beijing University of Aeronautics and Astronautics Press, 2007. 